\title{
Mobile Position Estimation based on Three Angles of Arrival using an Interpolative Neural Network
}

\author{
Omar Waleed Abdulwahhab \\ Computer Engineering Department, College of Engineering, \\ University of Baghdad
}

\begin{abstract}
In this paper, the memorization capability of a multilayer interpolative neural network is exploited to estimate a mobile position based on three angles of arrival. The neural network is trained with ideal angles-position patterns distributed uniformly throughout the region. This approach is compared with two other analytical methods, the average-position method which relies on finding the average position of the vertices of the uncertainty triangular region and the optimal position method which relies on finding the nearest ideal angles-position pattern to the measured angles. Simulation results based on estimations of the mobile position of particles moving along a nonlinear path show that the interpolative neural network approach outperforms the two other methods in its estimations for different noise conditions.
\end{abstract}

\section{General Terms}

Neural networks.

\section{Keywords}

Angle of arrival (AOA), average position, optimal position, interpolative neural network.

\section{INTRODUCTION}

Wireless mobile positioning approaches have received a significant attention in last years. The signal being received by a mobile is used to determine its location. The mobile signal is received from several base stations with known positions. Some characteristics of that signal, such as the strength of the signal, the time of arrival of the signal, or the angle of arrival of the signal are combined with the known positions of the base stations to determine the mobile position [1]. Each characteristic defines a locus on which the mobile station must lie. For multiple measurements, the point of intersection of the corresponding loci identifies the position of the mobile station [2].

There are mainly three techniques to determine the mobile position: received signal strength (RSS) from at least three base stations, time of arrival (TOA) from at least three base stations, time difference of arrival (TDOA) from at least three base stations, and angle of arrival (AOA) from at least two base stations.

From a geometrical point of view, to locate any point in a two dimensional space (plane) specifically, it is sufficient to know either its distance from three known points, its distance from a point and its direction with respect to a point (the same first point or another point), or its directions with respect to two points.

However, due to the possible existence of non-line-of-sight (NLOS) propagation errors and measurement errors, the intersection of multiple circles (for RSS and TOA techniques) may not yield a single common point. The result is usually an enclosed small area of uncertainty [3]. The same thing is true for angles of arrival (AOA) direction lines.

Several techniques were proposed to handle this issue. The simplest possible method is to estimate the location of the mobile station by choosing the center of the three intersections of the enclosed small area resulted from TOA measurements. In [3], Kalman filtering was used to process the sequences of mobile location measures obtained from time-of-arrival (TOA) geometric location techniques. [4] considered two methods: measured times of arrival (TOA) and angles of arrival (AOA). [5] presented a mobile station location method using constrained least-squares (CLS) estimation in the non-line-sight (NLOS) conditions. When some of the measurements are from NLOS paths, the location errors can be very large. The proposed method mitigates possible large TOA error measurements caused by NLOS. A location estimation scheme using fuzzy-based Interacting Multiple Model (IMM) smoother was proposed by [6]. It combines the time-of-arrival (TOA) and the received signal strength (RSS) measurements to achieve high location accuracy. The fuzzy technique was used to interpolate several linear equations to approximate the nonlinear RSS measurement. An approach of TOA (time of arrival) estimation was proposed by [7] for subscribers location in mobile communication systems. The correlation approach and a frequency domain de-convolution were used to convert the TOA (Time of Arrival) estimation problem to an estimation of frequency. By the use of the higher order accumulations of the measurement data, the noise was suppressed and the TOA estimation could be obtained correctly. [8] described a new method of time of arrival (TOA) estimation for OFDM (orthogonal frequency division multiplexing) signals in indoor multipath environments for application to location tracking systems based on time difference of arrival. The problem was to estimate the line of sight (LOS) time delay between the transmitter and the receiver when the intervening multipath channel was not known. A hybrid location scheme, which combined both the satellite-based and the network-based signals, was proposed in [9]. The proposed scheme utilizes the two-step Least Square method for estimating the threedimensional position (i.e. the longitude, latitude, and altitude) of the mobile devices. The Kalman filtering technique was exploited to both eliminate the measurement noises and to track the trajectories of the mobile devices. A fusion algorithm was employed to obtain the final location estimation from both the satellite-based and the networkbased systems. [10] designed a directional propagation model, the Modified Directional Propagation Model (MDPM), which makes use of a common signal propagation model to perform location estimation. It is a signal strength based algorithm which estimates the location of the Mobile Station by signal strength received from the nearly base stations. [11] illustrated 
hybrid proposed schemes that combine time of arrival (TOA) at three base stations and angle of arrival (AOA) at the serving base station to estimate a location of the mobile station. These schemes mitigate the NLOS effect by the weighted sum of the intersections between the TOA circles and the AOA line. An adaptive fuzzy logic estimator in a direct sequence code division multiple access (DS/CDMA) cellular system was proposed in [2]. It is based on the measured pilot signal strengths by the mobile station from several nearby base stations. A smoother that uses past and current output data from the fuzzy estimator was used to improve the accuracy of the location estimation. [12] combined the time difference of arrival (TDOA) measurements from the forward link pilot signals with the angle of arrival (AOA) measurement from the reverse link pilot signal. A two-step least square location estimator was developed based on a linear form of the AOA equation in the small error region. [13] proposed a location technique that estimates the line-of-sight (LOS) ranges based on NLOS range measurements. The approach utilizes a constrained nonlinear optimization approach for range measurements available from three base stations. The constraints were extracted from bounds on the NLOS error and the relationship between the true ranges. [14] proposed a method based on differences of downlink signal attenuations. This approach results in circles that the mobile station must lie on. The estimated mobile location is the point of intersection of these circles.

As stated in [15], there are convergence problems in the traditional Taylor series algorithm (TSA) and the hybrid lines of position algorithm (HLOP), and even if the measurements are accurate, the performance of these algorithms depends highly on the relative position of the mobile station and base stations. Therefore, they proposed an algorithm that combined both time of arrival (TOA) and angle of arrival (AOA) measurements to estimate the location of the mobile station in NLOS environments by utilizing the intersections of two circles and two lines and using the most resilient backpropagation (RPROP) neural network learning technique to estimate the location of the mobile station.

However, because of noisy measurements, it is preferable to collect data from multiple base stations in order to resolve the ambiguities resulted from multiple crossings of the lines of position and to improve the positioning accuracy [16].

\section{POSITION ESTIMATION BASED ON THREE ANGLES OF ARRIVAL}

Fig. 1 shows the mobile station MS and the three closest base stations, $\mathrm{BS}_{0}, \mathrm{BS}_{1}$, and $\mathrm{BS}_{2}$. The angles of arrival at the mobile station are $\alpha, \beta$, and $\gamma$ with respect to base stations $\mathrm{BS}_{0}, \mathrm{BS}_{1}$, and $\mathrm{BS}_{2}$, respectively.

Equation of $\overleftrightarrow{\left(\mathrm{BS}_{0}\right)(\mathrm{MS})}$ :

$y=y_{0}+(\tan \alpha)\left(x-x_{0}\right)$

(1)

Equation of $\overleftrightarrow{\left(\mathrm{BS}_{1}\right)(\mathrm{MS})}$ :

$y=y_{1}-(\tan \beta)\left(x-x_{1}\right)$

(2)

Equation of $\overleftrightarrow{\left(\mathrm{BS}_{2}\right)(\mathrm{MS})}$ :

$y=y_{2}+(\tan \gamma)\left(x-x_{2}\right)$

(3)

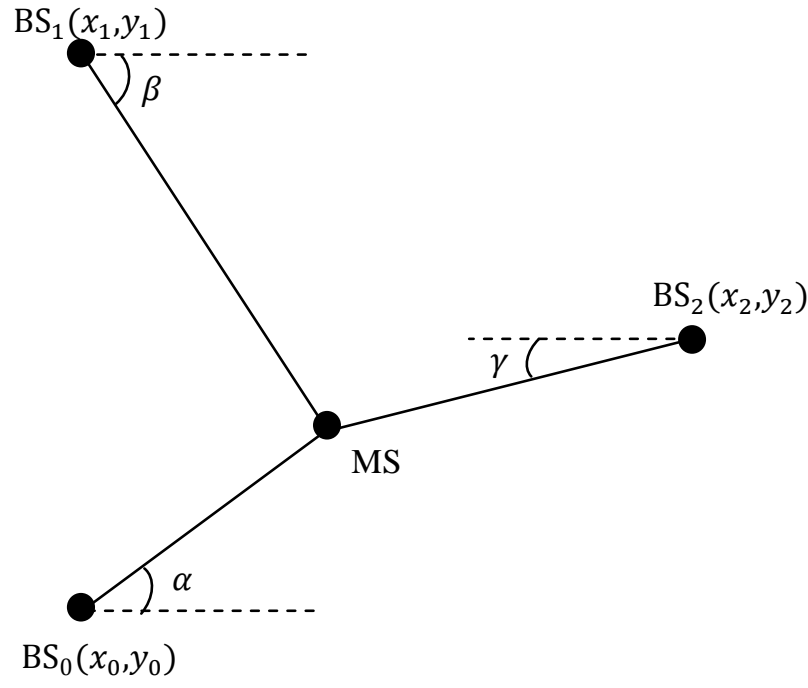

Fig. 1: Geometric layout of three angles of arrival approach

\section{AVERAGE POSITION METHOD}

Given the values of the three angles $\alpha, \beta$, and $\gamma$, the mobile station position can be determined by intersecting the three lines $\overleftrightarrow{\mathrm{BS}_{0} \mathrm{MS}}, \overleftrightarrow{\mathrm{BS}_{1} \mathrm{MS}}$, and $\overleftrightarrow{\mathrm{BS}_{2} \mathrm{MS}}$, i.e., finding the simultaneous solution $(x, y)$ of the three equations (1), (2), and (3). Ideally, the mobile station MS has consistent angles $\alpha, \beta$, and $\gamma$, with the base stations so that the simultaneous solution of any two equations of these three equations yields the same point. However, since the angles $\alpha, \beta$, and $\gamma$, subject to measurements errors, the three lines does not intersect at the same point. A reasonable method to estimate the mobile station position is to find the point of intersection of each two lines mutually and average the result.

1) Intersection of $\overleftrightarrow{\left(\mathrm{BS}_{0}\right)(\mathrm{MS})}$ and $\overleftrightarrow{\left(\mathrm{BS}_{1}\right)(\mathrm{MS})}$

$x_{a}=\frac{-y_{0}+y_{1}+(\tan \alpha) x_{0}+(\tan \beta) x_{1}}{\tan \alpha+\tan \beta}$

$y_{a}=y_{0}+(\tan \alpha)\left(x-x_{0}\right)$

(4)

2) Intersection of $\overleftrightarrow{\left(\mathrm{BS}_{0}\right)(\mathrm{MS})}$ and $\overleftrightarrow{\left(\mathrm{BS}_{2}\right)(\mathrm{MS})}$

$x_{b}=\frac{-y_{0}+y_{2}+(\tan \alpha) x_{0}-(\tan \gamma) x_{2}}{\tan \alpha-\tan \gamma}$

$y_{b}=y_{0}+(\tan \alpha)\left(x-x_{0}\right)$

(5)

3) Intersection of $\overleftrightarrow{\left(\mathrm{BS}_{1}\right)(\mathrm{MS})}$ and $\overleftrightarrow{\left(\mathrm{BS}_{2}\right)(\mathrm{MS})}$

$x_{c}=\frac{y_{1}-y_{2}+(\tan \beta) x_{1}+(\tan \gamma) x_{2}}{\tan \beta+\tan \gamma}$

$y_{c}=y_{1}-(\tan \beta)\left(x-x_{1}\right)$

(6)

Thus, the required point is

$(x, y)=\left(\frac{x_{a}+x_{b}+x_{c}}{3}, \frac{y_{a}+y_{b}+y_{c}}{3}\right)$

(7) 


\section{THE OPTIMAL POSITION METHOD}

Equations (1), (2), and (3) can be solved for $\alpha, \beta$, and $\gamma$, respectively in terms of $x$ and $y$.

$\alpha=\tan ^{-1} \frac{y-y_{0}}{x-x_{0}}$

(8)

$\beta=\tan ^{-1}\left(-\frac{y-y_{1}}{x-x_{1}}\right)$

(9)

$\gamma=\tan ^{-1} \frac{y-y_{2}}{x-x_{2}}$

(10)

Considering $(x, y)$ as parameters, equations (8), (9), and (10) represent a surface $S$ in the $\alpha \beta \gamma-$ space. Each point $(\alpha, \beta, \gamma)$ on the surface corresponds to a point $(x, y)$ in the $x y-$ plane. However, the remaining points that do not lie on the surface do no correspond to points $(x, y)$ in the $x y-$ plane. In this paper, a noise is added to input angles; therefore in general an input point $(\alpha, \beta, \gamma)$ does not lie on the surface.

For a certain input $\left(\alpha_{0}, \beta_{0}, \gamma_{0}\right)$, the optimization method relies on finding an absolute minimum for the cost function

$$
\begin{gathered}
f(x, y)=\left(\alpha_{0}-\tan ^{-1} \frac{y-y_{0}}{x-x_{0}}\right)^{2}+\left(\beta_{0}-\tan ^{-1}\left(-\frac{y-y_{1}}{x-x_{1}}\right)\right)^{2} \\
+\left(\gamma_{0}-\tan ^{-1} \frac{y-y_{2}}{x-x_{2}}\right)^{2}
\end{gathered}
$$

which is the square of the distance between the point $\left(\alpha_{0}, \beta_{0}, \gamma_{0}\right)$ and a general point $(\alpha, \beta, \gamma)$ on the surface $S$. This method corresponds to that given in [17] but for AOA approach instead of TOA.

\section{GENERALIZATION AND MEMORIZATION IN AN \\ INTERPOLATIVE ASSOCIATIVE \\ NEURAL NETWORK}

The interpolative associative neural network that was employed in this paper implements the mapping

$\phi: R^{3} \rightarrow R^{2}, \phi\left(\mathbf{z}^{(i)}\right)=\boldsymbol{d}^{(i)}, i=1, \ldots, P$

$$
\begin{aligned}
& \text { where } \\
& \mathbf{z}^{(i)}=\left[\left[\alpha^{(i)}, \beta^{(i)}, \gamma^{(i)}\right]^{T} \text {, the } i\right. \text { 'th stored stimuli, } \\
& \boldsymbol{d}^{(i)}=[x, y]^{T} \text {, the } i \text { 'th stored response. }
\end{aligned}
$$

In general, if $\boldsymbol{z}=\mathbf{z}^{(i)}+\boldsymbol{\delta}$ is presented to the neural network, the output is

$$
\phi\left(\mathbf{z}^{(i)}+\boldsymbol{\delta}\right)=\boldsymbol{d}^{(i)}+\boldsymbol{\varepsilon} \text { where } \lim _{\boldsymbol{\delta} \rightarrow \mathbf{0}} \boldsymbol{\varepsilon}=\mathbf{0} .
$$

The magnitude of the vector $\boldsymbol{\varepsilon}$ introduces the distinction between interpolative and heteroassociative neural network. If $\boldsymbol{\varepsilon}=\mathbf{0}$ for $0<\boldsymbol{\delta}<\boldsymbol{\delta}_{\boldsymbol{i}}$, it is a heteroassociative neural network, while if $\boldsymbol{\varepsilon} \neq \mathbf{0}$ for $0<\boldsymbol{\delta}<\boldsymbol{\delta}_{\boldsymbol{i}}$, it is an interpolative neural network. In this paper, generalization is required so that when a certain input that does not belong to the training pattern set is presented to the neural network, the output must be a reasonable interpolated vector. On the other hand, memorization is necessary since some input patterns have no reasonable output since they are a noisy version of the actual input vector. Here, the neural network must remember the input pattern that is closest to the distorted one, and then generalize (if this input pattern does not belong to the training set).

As is well known in such problems, over-fitting is must be avoided. However, in this paper, over-fitting was excluded by training the neural network with ideal patterns derived from mathematical relation between position and angles of arrival.

\section{ARCHITECTURE OF NEURAL NETWORK}

The neural network used in this paper is a multilayer neural network with one hidden layer. The input layer has three units which equal to the three angles of arrival at the mobile station, the hidden layer has one hundred units, and the output layer has two units which equal to the estimated coordinates $(x, y)$ of the mobile. The activation function of each neuron in the hidden layer is the unipolar sigmoid and the activation function of each neuron in the output layer is the identity function. Thus, the equation that describes this network is

$\boldsymbol{o}=\boldsymbol{\Psi}(\boldsymbol{W}(\boldsymbol{\Gamma}(\mathbf{V z})))$,

where

$\mathbf{z}=[\alpha, \beta, \gamma]^{T}$

$\boldsymbol{o}=[x, y]^{T}$

$\mathbf{V}$ is an $100 \times 4$ hidden layer weight-bias matrix

W is a $2 \times 101$ output layer weight-bias matrix

$\Gamma$ is a sigmoid matrix operator

$\boldsymbol{\Psi}$ is an identity matrix operator

\section{GENERATION OF TRAINING PATTERNS}

The training patterns are generated uniformly throughout the movement region. Ideal angles-position patterns are derived by finding the corresponding angles $\alpha, \beta$, and $\gamma$ for certain position $(x, y)$ using equations (8), (9), and (10). This procedure generates a set of training pattern associations $\left\{\left[\alpha^{(i)}, \beta^{(i)}, \gamma^{(i)}\right]^{T},\left[d_{x}^{(i)}, d_{y}^{(i)}\right]^{T}, i=1, \ldots, P\right\}$. In this paper, 400 training patterns was generated, i.e., $P=400$.

\section{NEURAL NETWORK TRAINING USING ONLINE BACKPROPAGATION ALGORITHM}

The neural network was trained using online backpropagation algorithm with the previously obtained four hundred training patterns. The weight matrices $\mathbf{V}$ and $\boldsymbol{W}$ were initialized randomly. The stopping criteria was chosen based on the RMS (Root Mean Square) value of the error, defined by

$$
E_{R M S}=\sqrt{\frac{1}{P} \sum_{i=1}^{P}\left(\boldsymbol{d}^{(i)}-\boldsymbol{o}^{(i)}\right)^{T}\left(\boldsymbol{d}^{(i)}-\boldsymbol{o}^{(i)}\right)}
$$

where

$P=400$, the number of training patterns,

$\boldsymbol{d}^{(i)}$ is the $i$ 'th desired stored response,

$\boldsymbol{o}^{(i)}$ is the $i$ 'th actual output.

Training of the neural network was stopped when $E_{R M S}$ reaches 2 . The learning rate was 0.0001 and the momentum term was 0.5 . After 9009 epochs, $E_{R M S}$ reached 3, as shown in Fig. 2. However, it needed another 12800 epochs so that $E_{R M S}$ dropped from 3 to 2, as shown in Fig. 3. 


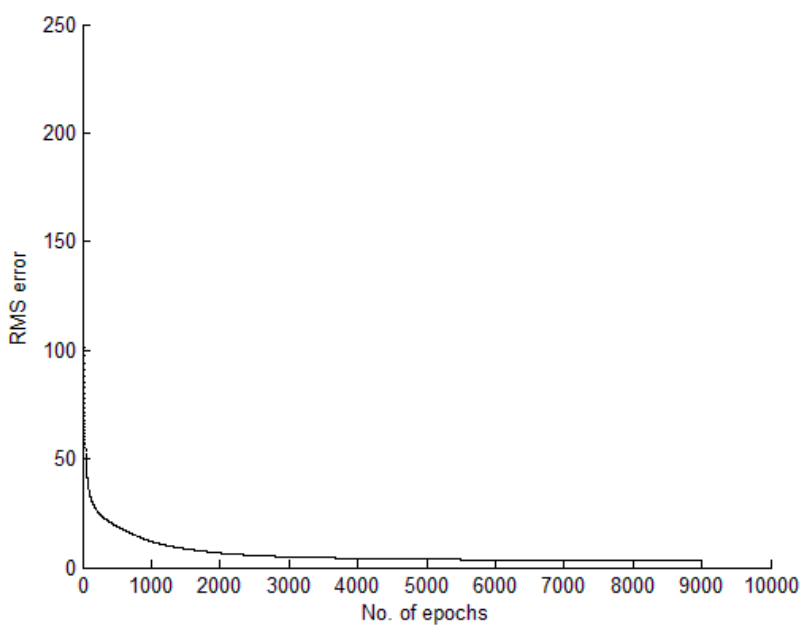

Fig. 2: $E_{R M S}$ versus number of epochs $\left(\operatorname{Min}\left(E_{R M S}\right)=3\right)$

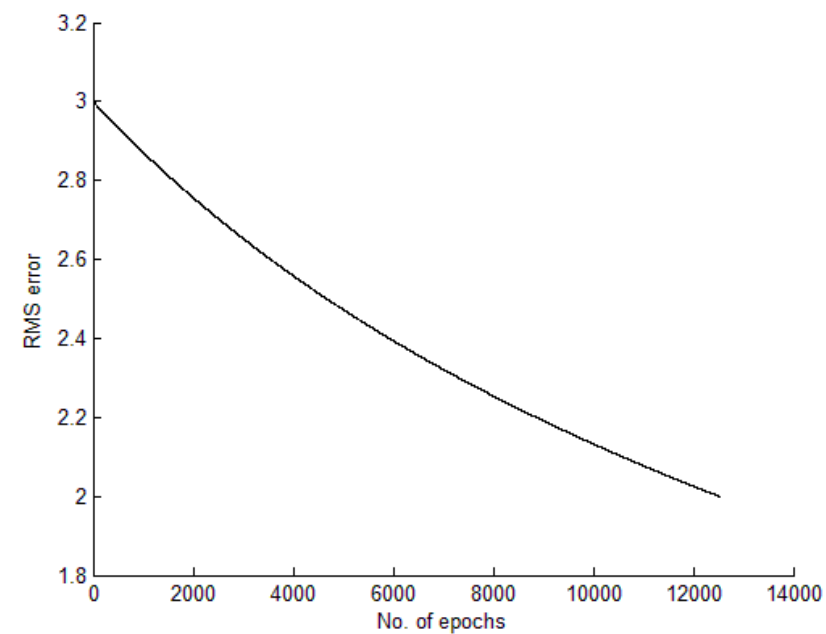

Fig. 3: $E_{R M S}$ versus number of epochs $\left(\operatorname{Min}\left(E_{R M S}\right)=2\right)$

\section{SIMULATION RESULTS}

The trained neural network was used to estimate the mobile position of a particle moving along a nonlinear path described by the equation $y=2 x^{3}+2 x^{2}+2 x+2$. The graph of this path is shown in Fig. 4. The neural network estimator was compared to the average position method and optimal position method. The comparison was splitted into two parts. Table 1 shows the results for the first part of the comparison for the neural network that was trained till $E_{R M S}=3$ and Table 2 shows the results for the second part of the comparison for the neural network that was trained so that $E_{R M S}$ reached 2. The comparisons were made for different angle measurement errors.

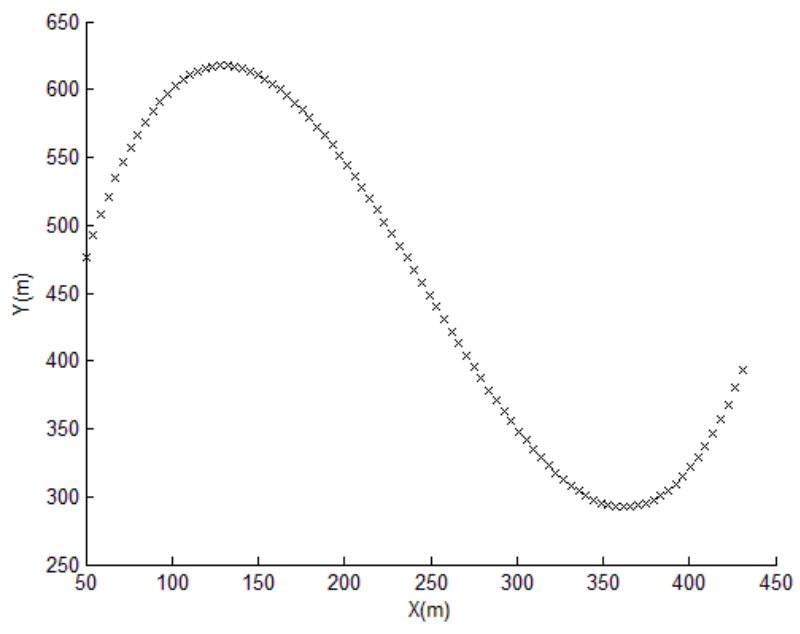

Fig. 4: The path traced by the particle

Table 1. Comparison between average position, optimal position, and neural network estimator with $E_{R M S}=3$

\begin{tabular}{|c|c|c|c|}
\hline $\begin{array}{c}\text { Error in } \\
\text { angles }\end{array}$ & $\begin{array}{c}\text { Average } \\
\text { position } \\
\text { RMS error } \\
(\mathrm{m})\end{array}$ & $\begin{array}{c}\text { Optimal } \\
\text { position } \\
\text { RMS error } \\
(\mathrm{m})\end{array}$ & $\begin{array}{c}\text { NN } \\
\text { estimator } \\
\text { RMS error } \\
(\mathrm{m})\end{array}$ \\
\hline $0 \%$ & 0 & 0 & 2.1624 \\
\hline $1 \%$ & 5.6590 & 5.7057 & 4.6182 \\
\hline $2 \%$ & 11.2516 & 11.2739 & 8.9022 \\
\hline $3 \%$ & 16.7796 & 16.6953 & 13.2665 \\
\hline $4 \%$ & 22.2447 & 21.9903 & 17.5806 \\
\hline $5 \%$ & 27.6484 & 27.1670 & 21.8131 \\
\hline $6 \%$ & 32.9919 & 32.2339 & 25.9498 \\
\hline $7 \%$ & 38.2765 & 37.1960 & 29.9849 \\
\hline $8 \%$ & 43.5034 & 42.0585 & 33.9197 \\
\hline $9 \%$ & 48.6735 & 46.8344 & 37.7611 \\
\hline $10 \%$ & 53.7878 & 51.5290 & 41.5211 \\
\hline $15 \%$ & 78.5546 & 73.9984 & 59.5973 \\
\hline $20 \%$ & 102.0557 & 95.4246 & 77.2914 \\
\hline
\end{tabular}

Table 2. Comparison between average position, optimal position, and neural network estimator with $E_{R M S}=2$

\begin{tabular}{|c|c|c|c|}
\hline $\begin{array}{c}\text { Error in } \\
\text { angles }\end{array}$ & $\begin{array}{c}\text { Average } \\
\text { position } \\
\text { RMS error } \\
(\mathrm{m})\end{array}$ & $\begin{array}{c}\text { Optimal } \\
\text { position } \\
\text { RMS error } \\
(\mathrm{m})\end{array}$ & $\begin{array}{c}\text { NN } \\
\text { estimator } \\
\text { RMS error } \\
(\mathrm{m})\end{array}$ \\
\hline $0 \%$ & 0 & 0 & 1.4290 \\
\hline $1 \%$ & 5.6590 & 5.7057 & 4.2408 \\
\hline $2 \%$ & 11.2516 & 11.2739 & 8.5666 \\
\hline $3 \%$ & 16.7796 & 16.6953 & 12.8521 \\
\hline $4 \%$ & 22.2447 & 21.9903 & 17.0335 \\
\hline $5 \%$ & 27.6484 & 27.1670 & 21.0949 \\
\hline $6 \%$ & 32.9919 & 32.2339 & 25.0319 \\
\hline $7 \%$ & 38.2765 & 37.1960 & 28.8473 \\
\hline $8 \%$ & 43.5034 & 42.0585 & 32.5500 \\
\hline $9 \%$ & 48.6735 & 46.8344 & 36.1531 \\
\hline $10 \%$ & 53.7878 & 51.5290 & 39.6723 \\
\hline $15 \%$ & 78.5546 & 73.9984 & 56.5569 \\
\hline $20 \%$ & 102.0557 & 95.4246 & 73.2065 \\
\hline
\end{tabular}

\section{CONCLUSIONS}

From the simulation results that was obtained and shown in Table 1 and Table 2, the following conclusions can be drawn: 
(1) When there is no error in the measured signal characteristics, the direct analytical methods (such as average position and optimal position methods) give exactly the actual position of the mobile station while the trained neural network approximates the mobile position with some error. (2) In the presence of measurement error (due to NLOS and noise) the performance of the analytical methods begins to degrade because of the presence of the region of uncertainty while the neural network begins to exploit its memorization and generalization capabilities to handle this region. Therefore, as the percentage error of the angles measurements increases, the neural network begins to outperform the geometrical methods. (3) For the two versions of the neural network $\left(E_{R M S}=3\right.$ and $E_{R M S}=2$ ), the second one outperforms the first as it is obvious. The reason is that continuously training the network does not encounter an over-fitting problem because it was trained with ideal training patterns.

\section{REFERENCES}

[1] Karray, F. O. and Silva, C. D. (2004) Soft Computing and Intelligent Systems Design. England: Pearson education.

[2] SHEN, X. MARK, J. W. and YE, J. (2002) Mobile Location Estimation in CDMA Cellular Networks by Using Fuzzy Logic. Wireless Personal Communications. [online] vol. 22(1). P. 57-70. Available from: www.ivsl.org [Accessed: 10th April 2014].

[3] Wann, C. and Chen, Y. (2002) Position Tracking and Velocity Estimation for Mobile Positioning Systems. InWireless Personal Multimedia Communications. vol. 1. p. 310-314. Available from: www.ivsl.org [Accessed: 10th April 2014].

[4] Caffery, J. and St"uber, G. L. (1998) Subscriber Location in CDMA Cellular Networks. IEEE Transactions on Vehicular Technology. [online] vol. 47(2/May). P. 406416. Available from: www.ivsl.org [Accessed: 10th April 2014].

[5] Wei, K. and Lenan, W. (2009) Constrained Least Squares Algorithm for TOA-Based Mobile Location under NLOS Environments. In- 5th International Conference on Wireless Communications, Networking and Mobile Computing. P. 1-4. Available from: www.ivsl.org [Accessed: 10th April 2014].

[6] Yang, C., Chen, B. and Liao, F. (2010) Mobile Location Estimation Using Fuzzy-Based IMM and Data Fusion. IEEE Transactions on Mobile Computing. [online] vol. 9(10/October). p. 1424-1436. Available from: www.ivsl.org [Accessed: 10th April 2014].

[7] LiuYing, Liang,Y., and Wang, S. (2000) Location Parameters Estimation in Mobile Communication Systems. In- Communication Technology proceeding. vol. 1. p. 261-268. Available from: www.ivsl.org [Accessed: 10th April 2014].

[8] Voltz, P. J. and Hernandez, D. (2004) Maximum Likelihood Time of Arrival Estimation for Real-Time Physical Location Tracking of $802.11 \mathrm{a} / \mathrm{g}$ Mobile Stations in Indoor Environments. In- Position Location and Navigation Symposium. p. 585-591. Available from: www.ivsl.org [Accessed: 10th April 2014].

[9] Chen, C. and Feng, K. (2005) Hybrid Location Estimation and Tracking System for Mobile Devices. InIEEE 61st Vehicular Technology Conference. vol. 4. p. 2648-2652. Available from: www.ivsl.org [Accessed: 10th April 2014].

[10] Zhou, J., Chu, K. M., and Ng, J. K. (2009) A Probabilistic Approach to Mobile Location Estimation within Cellular Networks. In- 15th IEEE International Conference on Embedded and Real-Time Computing Systems and Applications. P. 341-348. Available from: www.ivsl.org [Accessed: 10th April 2014].

[11] Chen, C., Su, S., and Lu, C. (2010) Geometrical Positioning Approached for Mobile Location Estimation. In- 2nd IEEE International Conference on Information Management and Engineering. p. 268-272. Available from: www.ivsl.org [Accessed: 10th April 2014].

[12] Cong, L. and Zhuang, W. (2002) Hybrid TDOA/AOA Mobile User Location for Wideband CDMA Cellular Systems. IEEE Transactions on Wireless Communications. [online] vol. 1(3/July). p. 439-447. Available from: www.ivsl.org [Accessed: 10th April 2014].

[13] Venkatraman, S., Caffery, J. and You, H. (2004) A Novel ToA Location Algorithm Using LoS Range Estimation for NLoS Environments. IEEE Transactions on Vehicular Technology. [online] vol. 53(5/September). p. 1515-1524. Available from: www.ivsl.org [Accessed: 10th April 2014].

[14] Lin, D. and Juang, R. (2005). Mobile Location Estimation Based on Differences of Signal Attenuations for GSM Systems. IEEE Transactions on Vehicular Technology. [online] vol. 54(4/July). p. 1447-1454 Available from: www.ivsl.org [Accessed: 10th April 2014].

[15] Chen, C. and Lin, J. (2011) Applying Rprop Neural Network for the Prediction of the Mobile Station Location. Sensors. [online] vol. 11(4). p. 4207-4230. Available from: www.ivsl.org [Accessed: 10th April 2014].

[16] Landolsi, M. A., Muqaibel, A. H. , Al-Ahmari, A. S. , Khan, H.-R. and Al-Nimnim, R. A. (2010). Performance Analysis of Time-of-Arrival Mobile Positioning in Wireless Cellular CDMA Networks. In- Bouras, C. J. (ed.). Trends in Telecommunications Technologies. [online] Available from: http://www.intechopen.com/books/trends-intelecommunications-technologies/performance-analysisof-time-of-arrival-mobile-positioning-in-wirelesscellular-cdma-networks. [Accessed: 10th April 2014].

[17] Liu, H. Darabi, H. Banerjee, P. and Liu, J. (2007) Survey of Wireless Indoor Positioning Techniques and Systems. IEEE Transactions on Systems, Man, and Cybernetics - Part C: Applications and Reviews. [online] vol. 37(6/November). Available from: www.ivsl.org [Accessed: 10th April 2014]. 